\title{
A Derivative-Based Fast Autofocus Method in Electron Microscopy
}

\author{
M.E. Rudnaya - H.G. ter Morsche · J.M.L. Maubach • \\ R.M.M. Mattheij
}

Published online: 12 August 2011

(C) The Author(s) 2011. This article is published with open access at Springerlink.com

\begin{abstract}
Most automatic focusing methods are based on a sharpness function, which delivers a real-valued estimate of an image quality. In this paper, we study an $L^{2}$-norm derivative-based sharpness function, which has been used before based on heuristic consideration. We give a more solid mathematical foundation for this function and get a better insight into its analytical properties. Moreover an efficient autofocus method is presented, in which an artificial blur variable plays an important role.

We show that for a specific choice of the artificial blur control variable, the function is approximately a quadratic polynomial, which implies that after the recording of at least three images one can find the approximate position of the optimal defocus. This provides the speed improvement in comparison with existing approaches, which usually require recording of more than ten images for autofocus. The new autofocus method is employed for the scanning transmission electron microscopy. To be more specific, it has been implemented in the FEI scanning transmission electron microscope and its performance has been tested as a part of a particle analysis application.
\end{abstract}

Keywords Autofocus/focusing - Sharpness function ·

Scanning transmission electron microscopy

\section{Introduction}

Consider an optical device, such as a photocamera, a telescope, or a microscope. An image obtained with the op-

M.E. Rudnaya $(\bowtie) \cdot$ H.G. ter Morsche · J.M.L. Maubach ·

R.M.M. Mattheij

Eindhoven University of Technology, Eindhoven,

The Netherlands

e-mail: m.rudnaya@tue.nl tical device depends on a given object's geometry, known as the object function, and the optical device defocus. The method of automatic defocus determination, such that the recorded image has the highest possible quality (the image is in-focus), is known as automatic focusing or autofocus method.

The existing autofocus methods used for various types of optical devices are usually based on a sharpness function, a real-valued estimate of the image's sharpness. For a through-focus series an ideal sharpness function should reach a single optimum (maximum or minimum, depending on the definition of the sharpness function) at the infocus image. Existing sharpness functions are based on image derivative [1, 23, 39], variance [4, 31], autocorrelation [9, 24, 29, 38], histogram [14, 40] or Fourier transform [11, $28,35,37]$. Overviews of existing sharpness functions can be found in [18, 28, 32, 40].

An autofocus method can be established in two different ways:

- A number of images is taken within a wide defocus range and for each image the sharpness function is computed giving a discrete set of sharpness function values. Then the optimal image (the in-focus image) is determined as the optimum of this discrete set of data (course focusing). Eventually the same procedure is repeated within a smaller defocus range around the optimum, found on the previous step (fine focusing).

- Starting out with an initial defocus parameter $d$, an iterative optimization method is used to find the optimal defocus value $d_{0}$ (for example, Fibonacci search [18, 40], Nelder-Mead simplex method [31] or Powell interpolation-based trust-region method [26]).

The first approach requires recording of about 20-30 images, which can be time-consuming for real-world applications. The goal of the second approach is to minimize the 
number of images necessary to perform the autofocus. It usually requires not less than 10 images for the autofocus procedure.

In literature a number of sharpness functions has been considered and discussed for various optical devices, such as photographic and video cameras [5, 11, 14], telescopes [12, 21], light microscopes [1, 9, 18, 32, 34, 39, 40] and electron microscopes [4, 24, 27, 28, 35, 37]. In this paper we use the electron microscopy as a reference application for our autofocus method. To be more precise, the experimental application is tested for low resolution scanning transmission electron microscopy (STEM).

For many practical applications in STEM, defocus has to be adjusted regularly during the continuous image recording process. For instance, in electron tomography 50-100 images are recorded at different tilt angles, where each tilting changes the defocus [37]. Other possible reasons for change in defocus are for instance the instabilities of the electron microscope and environment, as well as the magnetic nature of some samples. The capacity of the modern processors allow computations of a sharpness function within a negligible amount of time. However, image recording might require a noticeable amount of time. In particularly in STEM, one image recording can take 1 -to-10 seconds. For this reason the development of a method that requires fewer images is important. A number of methods implemented on aberratedcorrected electron microscopes are able to correct high and low aberrations including defocus [8, 19, 41]. For defocus correction these methods require from one to four images only. However, they are based on specific assumptions about the object geometry. These methods are not suitable for applications that require continuous operation since they are not fully autonomous [36]. In addition some of them make use of additional equipment, such as aberration correctors or a special camera, which is not a part of every microscope.

In this paper we study derivative-based sharpness functions. The advantage of using these functions has been already shown experimentally for scanning electron microscopy images $[27,28]$. Some of them are based on a $L^{2}$ norm of an image derivative [1, 14, 40]. The use of these functions is heuristic in nature. Usually they are based on the assumption that the in-focus image has a larger difference between neighboring pixels than the defocused one. In this paper we show analytically that for the noise-free image formation the $L^{2}$-norm derivative-based sharpness function reaches its optimum for the in-focus image, and does not have any other optima. Moreover under certain assumptions the function can accurately be approximated by a quadratic polynomial. The error of this approximation can be decreased by adjusting the artificial blur control variable, which is given as an input to the autofocus method. The proposed quadratic polynomial interpolation leads to a new autofocus method that requires recording of three or four im- ages only. The method is implemented in FEI STEM and is demonstrated for a real-world microscopy application.

The paper is set up as follows: Sect. 2 explains the image formation modeling used in this paper. Section 3 provides the definition of the derivative-based sharpness function, and explains the process of automated focusing. In Sect. 4 theoretical observations on derivative-based sharpness function are given. Subsequently Sect. 5 describes the quadratic interpolation of the sharpness function and the autofocus method. Numerical computations for experimental data obtained from a STEM FEI microscope are shown in Sect. 6. Section 7 presents the results of the on-line autofocus correction method implemented and running on a FEI STEM prototype. Finally Sect. 8 provides discussion on relationship between the mathematical theory and real-world applications.

\section{Modeling}

Usually an image is two-dimensional. However, for the simplification of our analysis we restrict the theoretical observations to a one-dimensional setting. If the objective lens of the optical device is rotationally symmetric, this restriction does not affect the real problem, because the twodimensional case in image formation is a repetition of the one-dimensional case in an orthogonal direction. Nevertheless, in our numerical experiments and the real-world application (Sects. 6-7) two-dimensional images are used. One of the experiments will correspond to the situation of the non-symmetric lens (for instance, the lens with astigmatism aberration).

The Fourier transform $\hat{f}$ of a function $f \in \mathrm{七}^{2}(\mathbb{R})$ plays a fundamental role in our analysis and modeling. It is defined as follows

$\hat{f}(\omega)=\int_{-\infty}^{\infty} f(x) e^{-\mathrm{i} \omega x} \mathrm{~d} x$,

where $x$ is a spatial coordinate and $\omega$ is a frequency coordinate.

Images for which our sharpness function will be computed are the output images $f$ of the so-called image formation model represented by Fig. 1. The object's geometry (or the object function) is denoted by $\psi$. The filter $\varrho_{\sigma}$ in Fig. 1 describes the point spread function of an optical device. The point spread function can accurately be approximated by a Lévi stable density function for a wide class of optical devices [2, 3, 10]. The Lévi stable density function is implicitly defined via its Fourier transform as follows

$\hat{\varrho}_{\sigma}(\omega):=e^{-\frac{\sigma^{2} \omega^{2} \beta}{2}}, \quad 0<\beta \leq 1$

The parameter $\beta$ in (1) depends on the optical device, and $\sigma$ in (1) is known as the width of the point spread function. It 


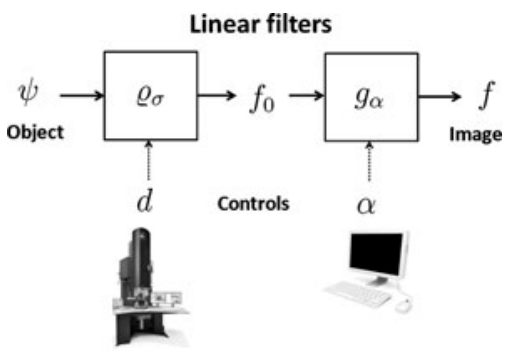

Fig. 1 The image formation model

is simply related to the control variable $d$, i.e. the defocus of the optical device

$\sigma=d-d_{0}$,

where $d_{0}$ is unknown. The goal of the autofocus procedure is to find the value of $d_{0}$.

The output of the $\varrho_{\sigma}$ filter is denoted by $f_{0}$ and often post-processed by a PC, cf. Fig. 1. In our model we assume that in such post-processing a Gaussian filter is applied to the image $f_{0}$

$g_{\alpha}(x):=\frac{1}{\sqrt{2 \pi} \alpha} e^{-\frac{x^{2}}{2 \alpha^{2}}}$.

Filtering with a Gaussian kernel is often applied for denoising purposes, which is an easy alternative to more advanced denoising techniques $[15,16,20]$. In our autofocus procedure the main use of the control variable $\alpha$ is not for denoising the image $f_{0}$. As explained in the following sections, it influences the approximation error when the sharpness function is replaced by a quadratic polynomial, but it does not change the location of $d_{0}$. The value of the control variable $\alpha$ is fixed during the autofocus process; i.e. when we attempt to find $d_{0}$ from a number of recorded images corresponding to different values of $d$ stemming from the same object function $\psi$.

We apply the linear image formation model, which is often used for various optical devices [2, 7, 23, 42], in particular for electron microscopes [4, 13]. This implies that the occurring filters are linear and space invariant which easily can be described by means of convolution products

$$
f_{0}:=\psi * \varrho_{\sigma}, \quad f:=f_{0} * g_{\alpha} .
$$

If no image post-processing is applied then, $\alpha=0$, and $f=f_{0}$.

\section{Sharpness Function and Problem Formulation}

In this section we introduce the derivative-based sharpness function explicitly and investigate its behavior with respect to the defocus parameter $d$. As the parameter $d$ is closely

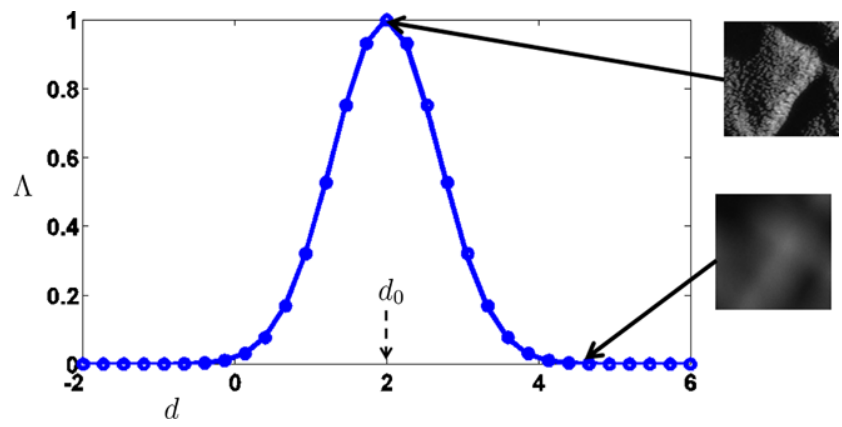

Fig. 2 Sharpness function $\Lambda$ reaches its optimum at the in-focus image. The goal of the autofocus procedure is to find the value of defocus $d_{0}$

related to the width $\sigma$ it is convenient to define the sharpness function as a function of $\sigma$ as (cf. $[14,18]$ )

$\Lambda(\sigma):=\left\|\frac{\partial}{\partial x} f\right\|_{L^{2}}^{2}$

For the linear image formation model (2), we have

$\Lambda(\sigma):=\left\|\frac{\partial}{\partial x}\left(\psi * \varrho_{\sigma} * g_{\alpha}\right)\right\|_{L^{2}}^{2}$.

Since $\sigma=d-d_{0}$, we will consider the function $\Lambda\left(d-d_{0}\right)$. For a through-focus series of images the sharpness function is computed at different values of $d$ for a fixed value of $\alpha$. A general behavior of a sharpness function is shown in Fig. 2. The image at the defocus $d=d_{0}$ is sharp or in-focus and the sharpness function reaches its optimum. The image far away from $d_{0}$ is called out-of-focus.

We recall that for our autofocus procedure $\alpha$ is fixed and a finite number, say $N$, of the defocus control $d$ are chosen: $d_{1}, \ldots, d_{N}$ with $d_{1}<d_{2}<\cdots<d_{N}$. For each of the corresponding images $f_{1}, f_{2}, \ldots, f_{N}$ the value of a sharpness function is computed

$\Lambda_{i}:=\Lambda\left(d_{i}-d_{0}\right), \quad i=1, \ldots, N$.

As already mentioned before, the problem of automated focusing is to estimate the optimum location $d_{0}$ of the sharpness function from the given points (5). The location $d_{0}$ is independent on the object function $\psi$.

In this paper our aim is to do this using a small number of recorded images, i.e., $N=3$ or $N=4$, while in other papers $N>10$ is usually used $[14,18,40,42]$. For this purpose we will look for the function shape which can accurately be approximated by a quadratic polynomial. In the next section the error estimates of such an approximation for derivativebased sharpness function are provided. 


\section{Theoretical Observations}

In this section we collect some useful properties of the derivative based sharpness function. First, in Sect. 4.1, we deal with general properties of $\Lambda$ in case the spread function $\varrho_{\sigma}$ is a Lévi stable density function. In Sect. 4.2, we restrict ourselves to the Gaussian point spread functions and study in more detail properties of $\Lambda$ for a typical collection of object functions: A Gaussian particle and the more general case of a digital image.

\subsection{General Properties of the Sharpness Function $\Lambda$}

Property 1 If $f$ is given by the linear image formation model (2) then the sharpness function $\Lambda$ is

$\Lambda(\sigma)=\frac{1}{2 \pi} \int_{-\infty}^{\infty} \omega^{2}|\hat{\psi}(\omega)|^{2} e^{-\sigma^{2} \omega^{2 \beta}} e^{-\alpha^{2} \omega^{2}} \mathrm{~d} \omega$.

Proof For $\hat{\psi}, \hat{g}, \hat{f}$, the Fourier transforms of $\psi, g, f$ respectively, it holds that $\hat{f}=\hat{\psi} \hat{\varrho}_{\sigma} \hat{g}_{\alpha}$. Then from Parseval's identity we find

$$
\begin{aligned}
\Lambda(\sigma) & :=\left\|\frac{\partial}{\partial x} f\right\|_{L^{2}}^{2}=\frac{1}{2 \pi}\|\omega \hat{f}\|_{L^{2}}^{2} \\
& =\frac{1}{2 \pi} \int_{-\infty}^{\infty} \omega^{2}|\hat{\psi}(\omega)|^{2}\left|\hat{\varrho}_{\sigma}(\omega)\right|^{2}\left|\hat{g}_{\alpha}(\omega)\right|^{2} \mathrm{~d} \omega .
\end{aligned}
$$

We assume that the object function satisfies the property

$$
\int_{-\infty}^{\infty}|\psi(x)| \mathrm{d} x<\infty
$$

which holds throughout the paper. In practice this property will be easily satisfied because the function $\psi$ has a finite domain, i.e., the image has a finite size. As a consequence we have that $\hat{\psi}$ is bounded and continuous. In case of a digital image (cf. Sect. 4.2) having a finite number of pixels, $\hat{\psi}$ is a tri-geometric polynomial which again is bounded and smooth.

Property 2 For the object function (7), the sharpness function $\Lambda(\sigma)$ is smooth, and is strictly increasing for $\sigma<0$ and strictly decreasing for $\sigma>0$.

Property 3 For the object function (7) and $\alpha>0$, the sharpness function $\Lambda(\sigma)$ has a finite maximum at $\sigma=0$

$$
\max _{\sigma} \Lambda(\sigma)=\Lambda(0)
$$

Properties 2-3 follow directly from (6). Figure 3 shows a numerically computed sharpness functions $\Lambda$ for different values of $\alpha$.

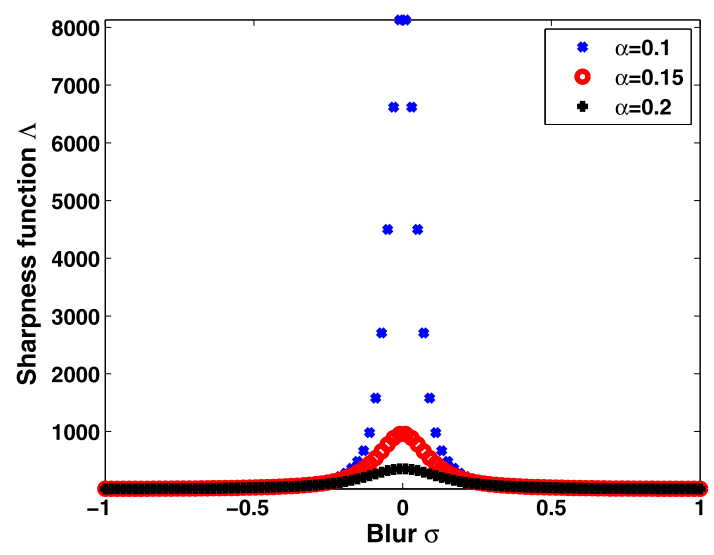

Fig. 3 Numerically computed sharpness functions $\Lambda$

Due to the physical limitations of the optical device the width of the point spread function has a positive underbound: $\sigma>\sigma_{0}$ for a certain positive number $\sigma_{0}$. From now on we consider a Gaussian point spread function, i.e. $\beta=1$ in (1). A Gaussian function (or a composition of Gaussian functions) is often used as an approximation of the point spread function for different optical devices [20, 23, 42], including the electron microscopes [4, 22].

As a benchmark case we deal with an object function for which the power spectrum corresponds to a Gaussian function. Such images often occur in experimental images from single particles. In our one-dimensional setting we may therefore assume that

$|\hat{\psi}(\omega)|^{2}=C e^{-\omega^{2} \gamma^{2}}, \quad C>0, \gamma \geq 0$.

For $\gamma=0$ in (8), $|\hat{\psi}|^{2}$ is a constant, which corresponds to the situation when the object is nearly amorphous [4].

Property 4 For the object function (8) and a Gaussian point spread function we have

$\Lambda(\sigma)=\frac{C}{4 \sqrt{\pi}\left(\sigma^{2}+\alpha^{2}+\gamma^{2}\right)^{\frac{3}{2}}}$.

Proof By substituting $\eta=\sqrt{\sigma^{2}+\alpha^{2}+\gamma^{2}}$ in the identity

$\int_{-\infty}^{\infty} \omega^{2} e^{-\eta^{2} \omega^{2}} \mathrm{~d} \omega=\frac{\sqrt{\pi}}{2 \eta^{3}}$

we obtain

$$
\begin{aligned}
\Lambda(\sigma) & =\frac{C}{2 \pi} \int_{-\infty}^{\infty} \omega^{2} e^{-\left(\sigma^{2}+\alpha^{2}+\gamma^{2}\right) \omega^{2}} \mathrm{~d} \omega \\
& =\frac{C}{4 \sqrt{\pi}\left(\sigma^{2}+\alpha^{2}+\gamma^{2}\right)^{\frac{3}{2}}} .
\end{aligned}
$$

We also observe that the location $d_{0}$ of the maximum of $\Lambda$ does not depend on $\alpha$. This, of course, will be true in general. 
Note that for the object function (8) the function

$F(d):=\Lambda^{-2 / 3}\left(d-d_{0}\right)$,

is a quadratic polynomial

$$
F(d)=\sqrt[3]{\frac{\pi}{C^{2}}}\left(\left(d-d_{0}\right)^{2}+\alpha^{2}+\gamma^{2}\right) .
$$

It will be shown that in the general case the function $F(d)$ can be well approximated by a quadratic polynomial for suitable choices of the blur variable $\alpha$. The quadratic shape of the function (10) makes finding its optimum faster and more robust in the real-world applications.

\subsection{Digital Image Object}

In classical signal analysis a discrete signal $\psi$ is modeled as a finite linear combination of delta functions (cf. [25])

$\psi(x)=\sum_{k=1}^{K} a_{k} \delta\left(x-\mu_{k}\right), \quad a_{k} \geq 0$.

In our setting, the finite sequence of numbers $a_{k}$ are the pixel values located at $x=\mu_{k}$ of the one-dimensional object function, $K$ is the number of pixels in the image. We consider an equally distributed set of pixels, so

$\mu_{k}:=k \tau, \quad \tau>0$

The sampling period $\tau$ is known as the pixel width. We define the vector of pixel values

$\mathbf{a}:=\left(a_{k}\right)_{k=1}^{K}$.

In this paper we consider the image with a finite number of pixels, i.e. $K<\infty$.

Proposition 1 The power spectrum of the object function (11) can be expressed as

$|\hat{\psi}(\omega)|^{2}=\sum_{m} \rho_{m} e^{\mathrm{i} m \tau \omega}$

where

$\rho_{m}:=\sum_{l} a_{l} a_{m+l}$

are the autocorrelation coefficients of the pixel values.

Proof The Fourier transform of the object function (11)

$\hat{\psi}(\omega)=\sum_{k} a_{k} \int_{-\infty}^{\infty} e^{-\mathrm{i} x \omega} \delta(x-k \tau) \mathrm{d} x=\sum_{k} a_{k} e^{-\mathrm{i} k \tau \omega}$ is a periodic function with the period $\frac{2 \pi}{\tau}$. Then its squared modulus $|\hat{\psi}(\omega)|^{2}$ is also a periodic function with period $\frac{2 \pi}{\tau}$ having the Fourier expansion

$|\hat{\psi}(\omega)|^{2}=\sum_{m} \rho_{m} e^{\mathrm{i} m \tau \omega}$,

where

$$
\begin{aligned}
\rho_{m} & =\frac{\tau}{2 \pi} \int_{-\frac{\pi}{\tau}}^{\frac{\pi}{\tau}}|\hat{\psi}(\omega)|^{2} e^{-\mathrm{i} m \tau \omega} \mathrm{d} \omega \\
& =\frac{\tau}{2 \pi} \int_{-\frac{\pi}{\tau}}^{\frac{\pi}{\tau}} \overline{\hat{\psi}(\omega)} \sum_{l} a_{l} e^{-\mathrm{i} l \tau \omega} e^{-\mathrm{i} m \tau \omega} \mathrm{d} \omega \\
& =\frac{\tau}{2 \pi} \sum_{l} a_{l} \int_{-\frac{\pi}{\tau}}^{\frac{\pi}{\tau}} \overline{\hat{\psi}(\omega)} e^{-\mathrm{i}(l+m) \tau \omega} \mathrm{d} u \\
& =\sum_{l} a_{l} \bar{a}_{m+l}=\sum_{l} a_{l} a_{m+l} .
\end{aligned}
$$

From definition (15) it trivially follows that

$\sum_{m} \rho_{m}=\|\mathbf{a}\|_{1}^{2}$

Property 5 The sharpness function $\Lambda$ can be expressed by means of the autocorrelation coefficients (15) as follows

$\Lambda(\sigma)=\frac{1}{2 \pi\left(\sigma^{2}+\alpha^{2}\right)^{3 / 2}} \sum_{m} \rho_{m} \int_{-\infty}^{\infty} \omega^{2} e^{\frac{\mathrm{i} m \omega \tau}{\sqrt{\alpha^{2}+\sigma^{2}}}} e^{-\omega^{2}} \mathrm{~d} \omega$.

Proof The proof is straightforward after we rewrite the sharpness function (6) for $\beta=1$ as

$$
\begin{aligned}
\Lambda(\sigma)= & \frac{1}{2 \pi\left(\sigma^{2}+\alpha^{2}\right)^{3 / 2}} \\
& \times \int_{-\infty}^{\infty} \omega^{2}\left|\hat{\psi}\left(\frac{\omega}{\sqrt{\alpha^{2}+\sigma^{2}}}\right)\right|^{2} e^{-\omega^{2}} \mathrm{~d} \omega .
\end{aligned}
$$

and substitute the expression for the power spectrum (14).

In the two propositions below we approximate the sharpness function $\Lambda$ by a function of the type $\frac{C}{\left(\alpha^{2}+\sigma\right)^{3 / 2}}$ in such a way that $\Lambda$ can be written as

$\Lambda(\sigma)=\frac{C}{\left(\alpha^{2}+\sigma^{2}\right)^{3 / 2}}(1+R(\sigma))$,

where $C$ depends only on the pixel values $C=C(\mathbf{a})$ and a relative error $R$, which can be small in typical circumstances. This implies that the function (10) can be expressed as

$F(d)=\mathcal{P}(d)(1+\epsilon(d))$, 
where $\mathcal{P}$ is a second order polynomial. For a small error $R(\sigma)$, the relative error $\epsilon(d)$ will be small: $\epsilon(d) \doteq-\frac{2}{3} R(\sigma)$.

In practical applications the value of $\sigma$ is important in relation to the pixel width $\tau$. For instance if $\sigma \gg \tau$, the image is totally out-of-focus (for example, Fig. 4(e)). It is often the case that $\sigma>\tau$, but not $\sigma \gg \tau$. However, by controlling the blur $\alpha$, the value $\sqrt{\alpha^{2}+\sigma^{2}}$ can be much larger than $\tau$, which is important for our error analysis in the next propositions.

Proposition 2 The sharpness function can be expressed as follows

$\Lambda(\sigma)=\frac{C_{1}}{2 \pi\left(\alpha^{2}+\sigma^{2}\right)^{3 / 2}}\left(1+R_{1}(\sigma)\right)$,

where

$\left|R_{1}(\sigma)\right| \leq K_{1} \frac{\tau}{\sqrt{\alpha^{2}+\sigma^{2}}}$,

and $C_{1}, K_{1}$ depend only on the pixel values $\mathbf{a}$.

Proof Splitting $e^{\frac{\mathrm{i} m \tau \omega}{\sqrt{\alpha^{2}+\sigma^{2}}}}$ into $\left(e^{\frac{\mathrm{i} m \tau \omega}{\sqrt{\alpha^{2}+\sigma^{2}}}}-1\right)+1$ in (17), one obtains

$$
\begin{aligned}
\Lambda(\sigma)= & \frac{1}{2 \pi\left(\sigma^{2}+\alpha^{2}\right)^{3 / 2}}(\underbrace{\int_{-\infty}^{\infty} \omega^{2} e^{-\omega^{2}} \mathrm{~d} \omega \sum_{m} \rho_{m}}_{C_{1}} \\
& \left.+\int_{-\infty}^{\infty} \omega^{2} e^{-\omega^{2}} \sum_{m} \rho_{m}\left(e^{\frac{\mathrm{i} m \tau \omega}{\sqrt{\alpha^{2}+\sigma^{2}}}}-1\right) \mathrm{d} \omega\right) .
\end{aligned}
$$

Applying (16), and (9) for $\eta=1$, one obtains

$C_{1}:=\int_{-\infty}^{\infty} \omega^{2} e^{-\omega^{2}} \mathrm{~d} \omega \sum_{m} \rho_{m}=\frac{\sqrt{\pi}}{2}\|\mathbf{a}\|_{1}$.

To estimate $R_{1}$ observe that

$\left|e^{\mathrm{i} \eta}-1\right|=2\left|\sin \frac{\eta}{2}\right| \leq|\eta|, \quad \eta \in \mathbb{R}$,

for $\eta=\frac{m \tau \omega}{\sqrt{\alpha^{2}+\sigma^{2}}}$, and consequently

$$
\left|\sum_{m} \rho_{m}\left(e^{\frac{\mathrm{i} m \tau \omega}{\sqrt{\alpha^{2}+\sigma^{2}}}}-1\right)\right| \leq\left(\sum_{m}|m| \rho_{m}\right) \frac{|\omega| \tau}{\sqrt{\alpha^{2}+\sigma^{2}}} .
$$

From the estimate (23) and $\int_{-\infty}^{\infty}|\omega|^{3} e^{-\omega^{2}} \mathrm{~d} \omega=1$ it follows that

$$
\begin{aligned}
& \left|\int_{-\infty}^{\infty} \omega^{2} e^{-\omega^{2}} \sum_{m} \rho_{m}\left(e^{\frac{\mathrm{i} m \tau \omega}{\sqrt{\alpha^{2}+\sigma^{2}}}}-1\right) \mathrm{d} \omega\right| \\
& \quad \leq\left(\sum_{m}|m| \rho_{m}\right) \frac{\tau}{\sqrt{\alpha^{2}+\sigma^{2}}} .
\end{aligned}
$$

Then the statement of the proposition is straightforward with

$K_{1}:=\frac{2}{\sqrt{\pi}} \frac{\sum_{m}|m| \rho_{m}}{\sum_{m} \rho_{m}}$

in (20).

It follows from the proposition that the function (10) can approximated by a quadratic polynomial at any accuracy by increasing the value of the blur $\alpha$.

Now let $\sigma \leq \tau$. It means that the image is almost in-focus and might be unsharp only slightly. Figures 4(a)-4(c) show the examples of artificially blurred images. From left to right: original image, blurred image with $\sigma / \tau=0.5$, blurred image with $\sigma / \tau=1$. We can hardly see any difference between original and blurred images. However, if we zoom into the details (Figs. 5(a)-5(c)) the difference is visible. This correspond to the fine focusing, which is considered in the proposition below.

Proposition 3 The sharpness function can be expressed as follows

$\Lambda(\sigma)=\frac{C_{2}}{2 \pi\left(\alpha^{2}+\sigma^{2}\right)^{3 / 2}}\left(1+R_{2}(\sigma)\right)$,

where

$\left|R_{2}(\sigma)\right| \leq K_{2} \frac{\alpha^{2}+\sigma^{2}}{\tau^{2}}$,

and $C_{2}, K_{2}$ depend only on the pixel values $\mathbf{a}$.

Proof Splitting $\sum_{m} \rho_{m}$ into $\rho_{0}+\sum_{m \neq 0} \rho_{m}$ in (17) one obtains

$$
\begin{aligned}
\Lambda(\sigma)= & \frac{1}{2 \pi\left(\sigma^{2}+\alpha^{2}\right)^{3 / 2}}(\underbrace{\rho_{0} \int_{-\infty}^{\infty} \omega^{2} e^{-\omega^{2}} \mathrm{~d} \omega}_{C_{2}} \\
& \left.+\sum_{m \neq 0} \rho_{m} \int_{-\infty}^{\infty} \omega^{2} e^{-\frac{\mathrm{i} m \tau \omega}{\sqrt{\alpha^{2}+\sigma^{2}}}} e^{-\omega^{2}} \mathrm{~d} \omega\right),
\end{aligned}
$$

$C_{2}:=\rho_{0} \int_{-\infty}^{\infty} \omega^{2} e^{-\omega^{2}} \mathrm{~d} \omega=\frac{\sqrt{\pi}}{2}\|\mathbf{a}\|_{2}$.

To estimate $R_{2}$ observe that

$\left|\int_{-\infty}^{\infty} \omega^{2} e^{-\omega^{2}} e^{\mathrm{i} \eta \omega} \mathrm{d} \omega\right|=\left|\frac{\sqrt{\pi}}{4}\left(2-\eta^{2}\right) e^{-\frac{\eta^{2}}{4}}\right| \leq \frac{4}{\eta^{2}}$, 


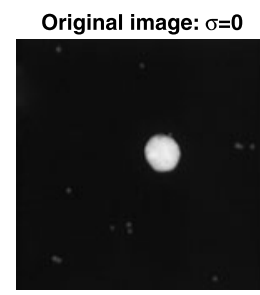

(a)

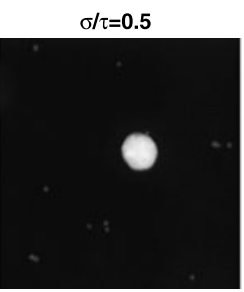

(b)

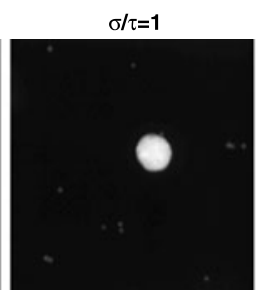

(c)

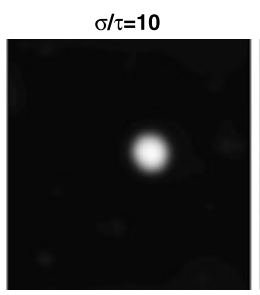

(d)

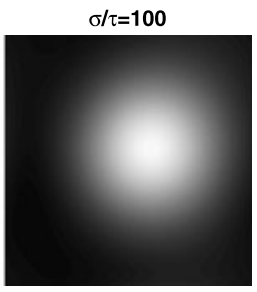

(e)

Fig. 4 Artificially blurred images of a gold particle with different values of $\sigma / \tau$

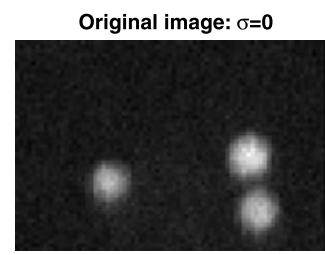

(a)

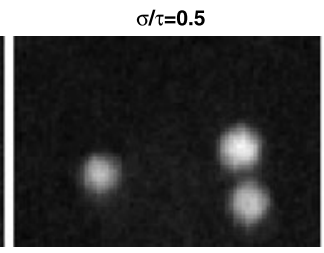

(b)

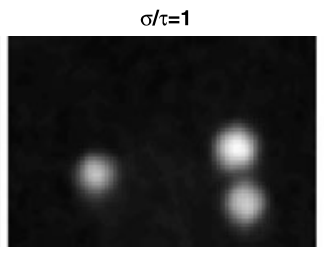

(c)

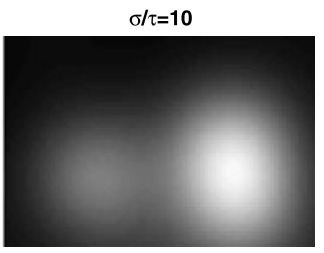

(d)

Fig. 5 Artificially blurred images of a gold particle with different values of $\sigma / \tau$. The images are the magnified versions of those shown in Fig. 4 . Only if we zoom into the small particles we can see the difference in the image quality for the small values of $\sigma$

i.e. substitute $\eta=\frac{m \tau}{\sqrt{\alpha^{2}+\sigma^{2}}}$

$\left|\int_{-\infty}^{\infty} \omega^{2} e^{-\omega^{2}} e^{\frac{\mathrm{i} m \tau \omega}{\sqrt{\alpha^{2}+\sigma^{2}}}} \mathrm{~d} \omega\right| \leq 4 \frac{\alpha^{2}+\sigma^{2}}{m^{2} \tau^{2}}$.

Then the statement of the proposition is straightforward with

$K_{2}:=\frac{8}{\sqrt{\pi}} \frac{\sum_{m \neq 0} \frac{\rho_{m}}{m^{2}}}{\rho_{0}}$

in $(25)$.

Proposition 3 considers the situation of a very fine focusing, which is different from Proposition 2, where a more general case is considered. However, it is shown that in both situations the function $F$ can be approximated by a quadratic polynomial with a given accuracy by means of adjusting the value of the control variable $\alpha$. This coincides with findings of Property 4 for a different object function model.

\section{The Autofocus Algorithm}

It has been mentioned before that the function evaluations in our problem are very expensive and derivative information is not available. For this reason quadratic interpolation is a convenient approach for computing a quadratic polynomial approximation of the function $F$. In our autofocus method we take the minimum of the polynomial as the minimum of the sharpness function. For the given data points $F_{k}:=F\left(d_{k}\right), k=1,2,3$ we interpolate the function $F$ by a polynomial $\mathcal{P}(d):=c_{0}+c_{1} d+c_{2} d^{2}$. So one has

$F(d)=\mathcal{P}(d)(1+\epsilon(d))$,

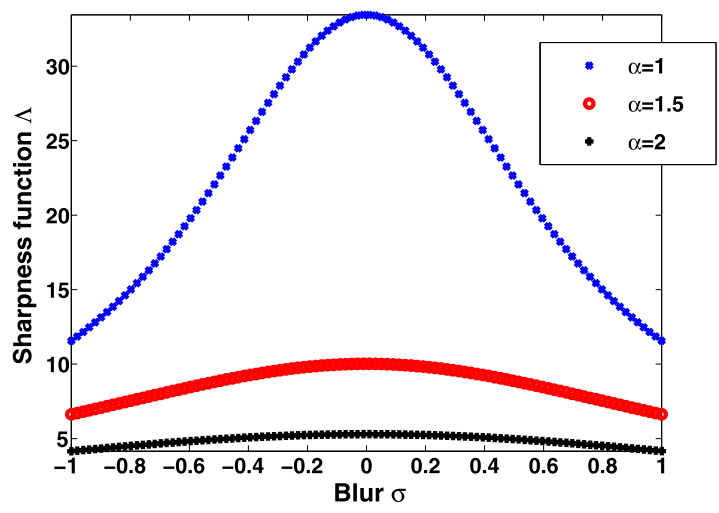

Fig. 6 Sharpness function $\Lambda$ computed for different values of the blur $\alpha$

where $P\left(d_{k}\right)=F_{k}, k=1,2,3$.

From Proposition 2 we conclude that the error $\epsilon(d)$ can be decreased by increasing $\alpha$. Theoretically the error of this approximation (cf. Proposition 2) can be made as small as needed by dramatically increasing the value $\alpha$. However, if $\alpha \rightarrow \infty$ then $F(d) \rightarrow 0$ and all its derivatives, which may causes numerical errors and make it difficult to find the optimum of the function. Figure 6 shows three functions (10) computed for different $\alpha$ values. In the next section it will be shown how the large values of $\alpha$ influence the shape of the function computed for experimental through-focus series.

In the autofocus algorithm below we use the parameter $N_{\text {max }}$, which corresponds to the maximum number of images we would like to record for the autofocus. The primary goal of this paper is to find the in-focus image after recording of 3 or 4 images only. However, in some practical 
applications, where the higher precisions are required, the recording of more images could be helpful.

\section{Autofocus algorithm:}

1. Let $d_{2}$ be the current defocus control value of the optical device. Choose a $\Delta d$, then $d_{1}:=d_{2}-\Delta d, d_{3}:=d_{2}+$ $\Delta d$.

2. Obtain three images at $d_{1}, d_{2}, d_{3}$ and compute $F_{1}, F_{2}, F_{3}$. We set $N=3$.

3. We fit $N$ given points with a quadratic polynomial $\mathcal{P}(d)$, and estimate the sharpness function optimum

$d_{N+1}=-\frac{c_{1}}{2 c_{2}}$

as the optimum of the quadratic polynomial. For $N=3$ this is the straightforward computation. For $N>3$ we obtain the overdetermined system

$$
\underbrace{\left(\begin{array}{ccc}
1 & d_{1} & d_{1}^{2} \\
\cdots & \ldots & \ldots \\
1 & d_{N} & d_{N}^{2}
\end{array}\right)}_{\mathrm{D}} \underbrace{\left(\begin{array}{c}
c_{0} \\
c_{1} \\
c_{2}
\end{array}\right)}_{\mathbf{c}}=\underbrace{\left(\begin{array}{c}
F_{1} \\
\ldots \\
F_{N}
\end{array}\right)}_{\mathbf{s}},
$$

for which a usual least squares solution $\mathbf{c}$ can be found in a regular way by solving the system

$$
\mathbf{D}^{T} \mathbf{D c}=\mathbf{D}^{T} \mathbf{s} .
$$

4. If $N=N_{\text {max }}$, stop. If for the given tolerance $d_{t o l} \in$ $\mathbb{R},\left|d_{N}-d_{N+1}\right|<d_{t o l}$, stop. Else, compute $F_{N+1}=$ $F\left(d_{N+1}\right)$ and go to the previous step.

It is important to note that only the last two steps of the described algorithm will be repeated interactively. Steps one and two are performed only ones, in the beginning of correction. The parameter $d_{t o l}$ can be determined from the knowledge of the optical device behavior. For instance, in electron microscopy the tolerable defocus error is defined as [37]

$d_{t o l}:=\sqrt{\left(\frac{w}{2}\right)^{2}+\left(\frac{t}{2}\right)^{2}}$,

where $t$ is the object's thickness and $w$ is the depth of field defined in [6] as

$w:=\frac{\tau}{\phi}$

where $\phi$ is the convergence semiangle of the magnetic lens and $\tau$ is the pixel width. The tolerable defocus error can be considered as the lower bound set by the depth of field

$d_{t o l}=\frac{\tau}{2 \phi}$

In the step three different numerical method could be used. The choice of the method is not quite significant for
Table 1 Overview of carbon cross grating experimental focus series

\begin{tabular}{llllll}
\hline$N$ & Magnification & $\begin{array}{l}\text { Pixel } \\
\text { width } \\
\tau[\mathrm{nm}]\end{array}$ & $\begin{array}{l}\text { Defocus } \\
\text { range } \\
\left(d_{N}-d_{1}\right)[\mathrm{nm}]\end{array}$ & $\begin{array}{l}\text { Defocus } \\
\text { step } \\
\Delta d[\mathrm{~nm}]\end{array}$ & $\begin{array}{l}\text { Number } \\
\text { of images }\end{array}$ \\
\hline 1. & $10000 \times$ & 42 & 36000 & 2000 & 19 \\
2. & $10000 \times$ & 42 & 10000 & 500 & 21 \\
3. & $200000 \times$ & 2.1 & 20000 & 1000 & 21 \\
4. & $200000 \times$ & 2.1 & 10000 & 500 & 21 \\
5. & $400000 \times$ & 1.05 & 900 & 50 & 19 \\
\hline
\end{tabular}

Table 2 Overview of gold particles experimental focus series

\begin{tabular}{llllll}
\hline$N$ & Magnification & $\begin{array}{l}\text { Pixel } \\
\text { width } \\
\tau[\mathrm{nm}]\end{array}$ & $\begin{array}{l}\text { Defocus } \\
\text { range } \\
\left(d_{N}-d_{1}\right)[\mathrm{nm}]\end{array}$ & $\begin{array}{l}\text { Defocus } \\
\text { step }\end{array}$ & $\begin{array}{l}\text { Number } \\
\text { of images }\end{array}$ \\
& & 42 & 31500 & 450 & 70 \\
\hline 1. & $10000 \times$ & 42 & 96 & 50 \\
2. & $10000 \times$ & 42 & 4704 & 16 & 51 \\
3. & $56000 \times$ & 7.5 & 800 & 80 & 71 \\
4. & $56000 \times$ & 7.5 & 5600 & 40 & 71 \\
5. & $115000 \times$ & 3.75 & 2800 & & \\
\hline
\end{tabular}

$N=4$. On-line experiments with the method implemented on a prototype FEI STEM for $N=3,4$ are presented in the following section.

\section{Numerical Experiments with STEM Images}

Ten experimental through-focus series are obtained with the FEI STEM microscope. Two different samples are used: a carbon cross grating sample and a gold particle sample. Carbon cross grating is the standard sample for STEM calibration. The gold particle sample is a typical image example, used for particle analysis applications. The size of each image in the series is $512 \times 512$ pixels. The series are obtained at different magnifications and with different defocus steps. Figures 7-8 show the first image in the series, the in-focus image, and the computed function $F$ values plotted versus the values of defocus control. Each of the figures represent five series, described in Tables 1-2 (carbon cross grating sample and gold particles sample correspondingly). The line numbers in the tables $N=1,2,3,4,5$ correspond to the columns of Figs. 7-8 (from left to right). For each series two functions are computed: with $\alpha=0$ (dotted line) and with $\alpha>0$ (dashed line). The values of both functions are scaled between 0 and 1 . Computed functions $F$ with $\alpha>0$ can accurately be approximated by a quadratic polynomial.

The series shown in the second columns of Figs. 7-8 are recorded with a small defocus step. The qualities of the first image in the series and the in-focus image do not differ so 

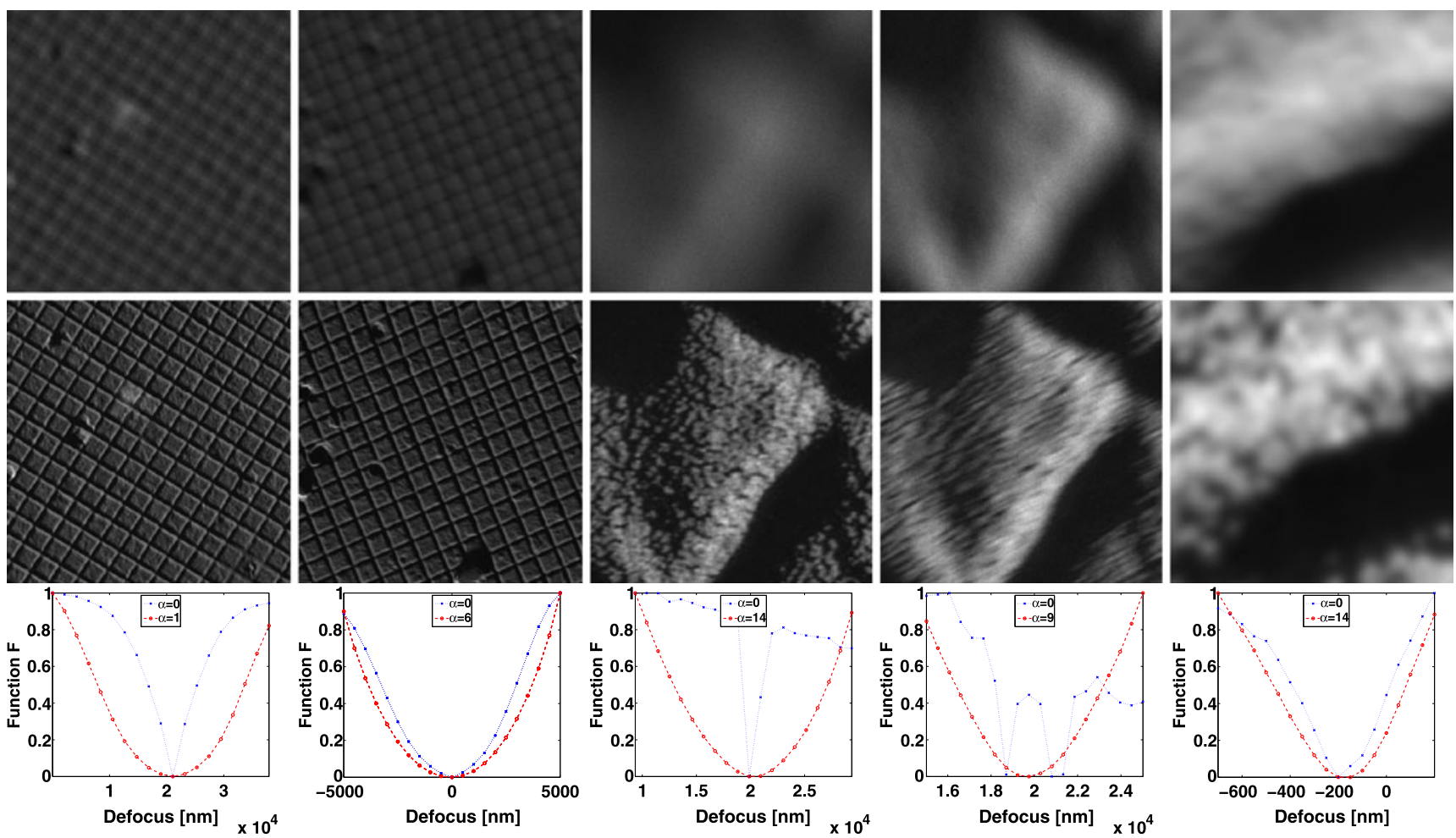

Fig. 7 Functions (10) computed for experimental STEM focus series of carbon cross grating sample. From top to bottom: The first image in the series, in-focus image from the series, functions with and without artificial blur plotted versus defocus. From left to right: Five different experimental focus series

much: We can see the details on the first images from the series, only the edges are a bit unsharp. It is shown in Tables 1-2 $(N=2)$ that these series have relatively small defocus ranges and defocus steps for particular magnification. For these cases the function $F$ has a shape nearly quadratic even with $\alpha=0$, as follows from Proposition 3. The function $F$ shape is different in a broader defocus range for the same sample at the same magnification (Fig. 7, first column and Fig. 8, first column). The functions with $\alpha=0$ have shapes similar to a Gaussian, but not a quadratic polynomial. In this case the functions have a nearly quadratic shape after applying the blur $\alpha$ to the images.

The fourth series of carbon cross grating (Fig. 7, fourth column) is the only experimental series recorded with the presence of astigmatism aberration. For other experimental series astigmatism of the magnetic lens has been corrected before the recording. The lens with astigmatism is not perfectly symmetric and as a consequence has more than one focal point [24], which results in the asymmetry of the point spread function. Consequently the recorded image cannot be totally sharp. The sharpness function might have local optima due to the presence of astigmatism [4, 31]. Two local minima can be seen in the plot. They disappear after applying the artificial blur. The influence of the astigmatism aberration on the derivative-based sharpness function, and the stigmatic sharpness function improvement after applying the artificial blur have been studied in [30].

In the last experiments (Figs. 7-8, fifth column) the magnification of the microscope is higher and as a consequence the influence of noise on the image quality increases. We can see that in these cases the blur $\alpha$ helps to cope with the noise in the function.

Figure 9 shows functions $F$ computed for different values of the blur $\alpha$ for experimental through-focus series of gold particles $(N=1,2)$. For the large $\alpha=20$ the function becomes noisy (not because of the noise in the date, but due to numerical errors) and does not provide useful information anymore. It follows that for the proper work of the method we have to make a proper choice of the value $\alpha$, which is not too large, but also not too small. This choice might depend on the sample geometry as well as on the level of noise and possible presence of astigmatism in the images. It also depends on the ratio of the given magnification (or the pixel width) to the defocus range. The choice does not have to be made every time, but once for a particular application, where we deal with the class of geometrical objects and microscope settings. The examples shown in Fig. 9 imply that $\alpha=2$ might be enough. However, Fig. 9 only shows the functions computed for the series of images recorded at the magnification $10000 \times$. For the higher magnifications the higher levels of noise are present in the images. In this case 

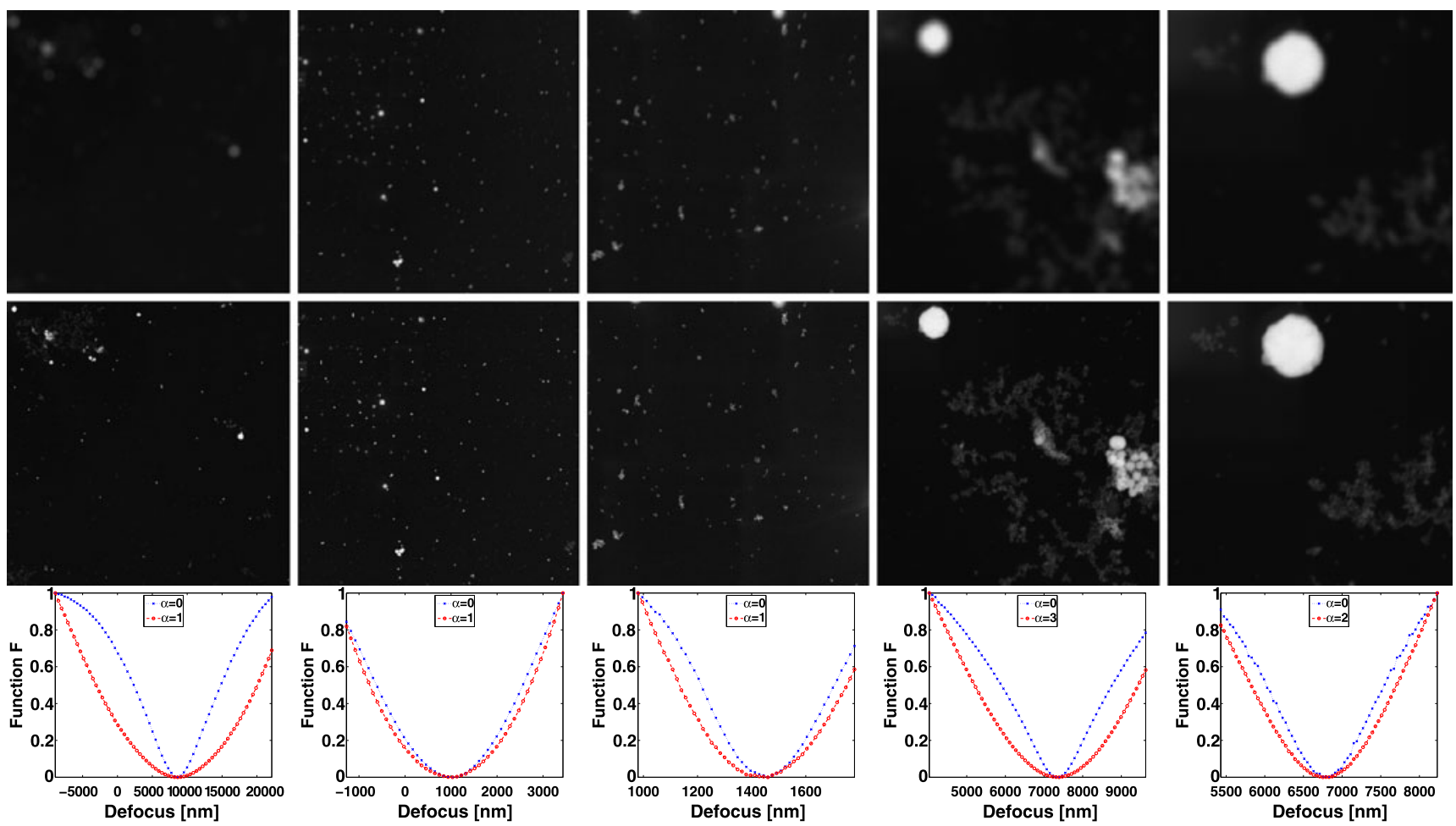

Fig. 8 Functions (10) computed for experimental STEM focus series of gold particles sample. From top to bottom: The first image in the series, in-focus image from the series, functions with and without artificial blur plotted versus defocus. From left to right: Five different experimental focus series

Fig. 9 Sharpness function $F$ computed for different values of the blur $\alpha$ for experimental through-focus series of gold particles $(N=1,2)$, Table 2
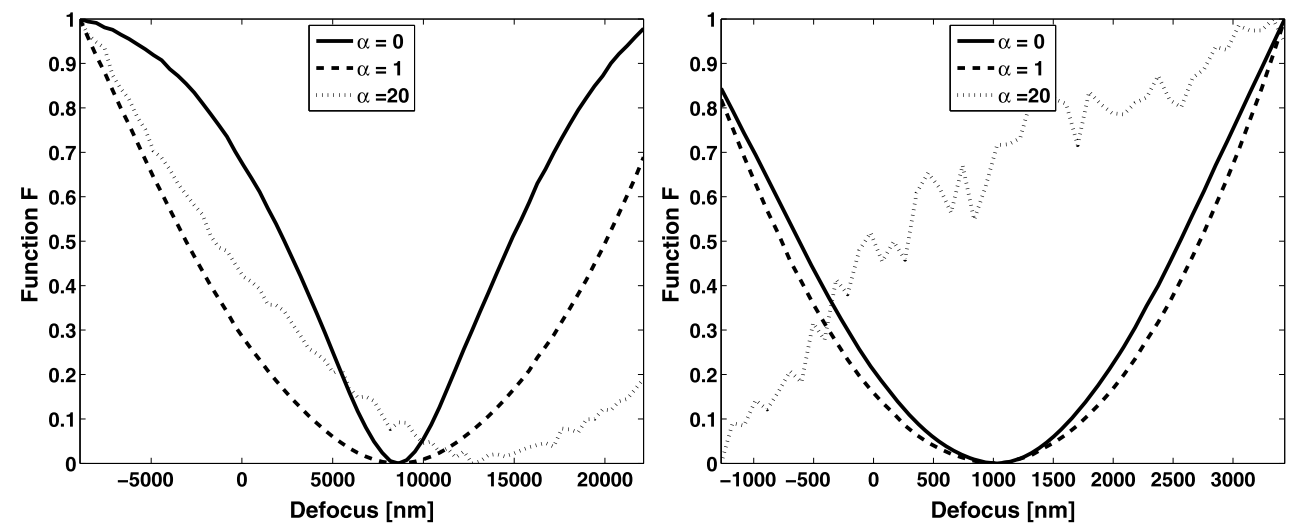

a higher value of $\alpha$ is needed (see for instance Fig. 8, where $\alpha=3$ is used).

\section{Application}

The method is tested in a prototype FEI Tecnai F20 STEM electron microscope. The Gaussian function provides only a rough approximation of the real-world STEM point spread function. A more accurate model based on the wave aberration function [13], which is less convenient for the analytical observations.
The tests are performed with the help of Java-based experimental platform (called EXPLA), which consists of a core that connects to the TEMScripting interface for FEI microscope control, and an application control framework [31]. For our experiment the autofocus method is implemented in Matlab V7.5 (R2007b). The method is integrated with a particle analysis application. The goal of this application is a statistical analysis of the particle distribution (particle locations and sizes). During the application run the images for further analysis are recorded at different positions and magnifications. During the run the position of the ideal defocus $d_{0}$ changes as the result of machine controls changes (stage 


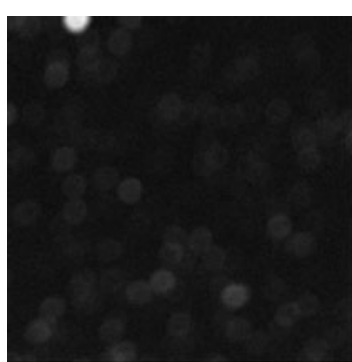

(a) $d_{1}=1447 \mathrm{~nm}$

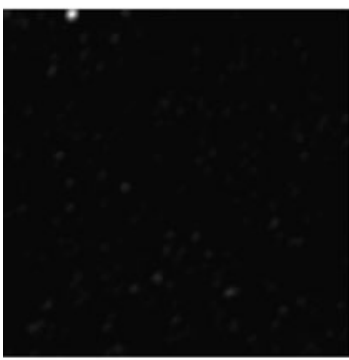

(e) $d_{2}=2447 \mathrm{~nm}$

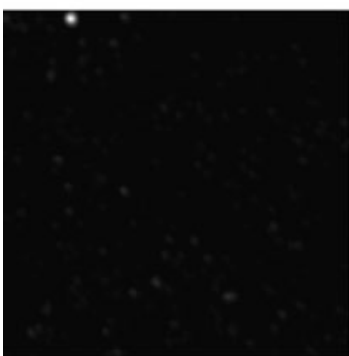

(i) $d_{3}=3447 \mathrm{~nm}$

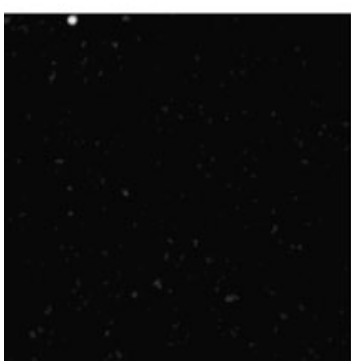

(m) Improved image $d_{4}=2961 \mathrm{~nm}$

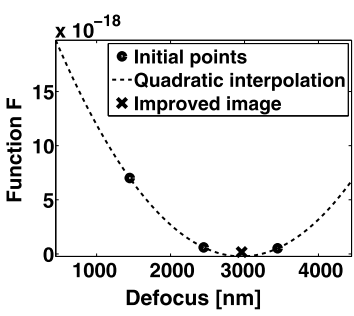

(q)

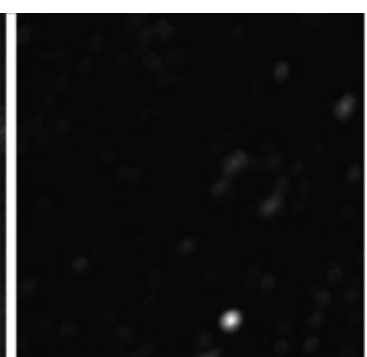

(b) $d_{1}=2451 \mathrm{~nm}$

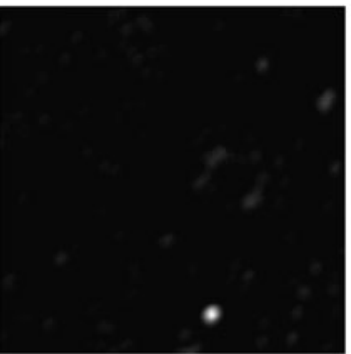

(f) $d_{2}=2576 \mathrm{~nm}$

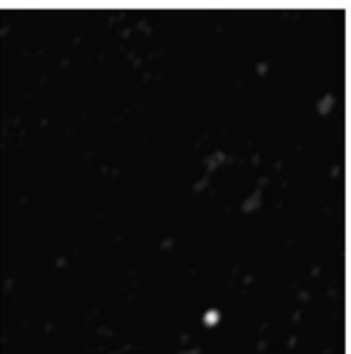

(j) $d_{3}=2701 \mathrm{~nm}$

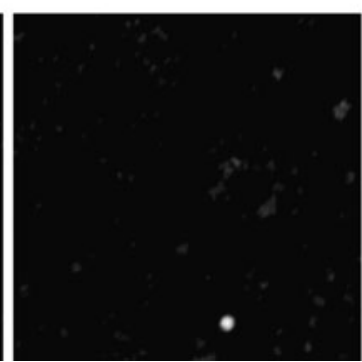

(n) Improved image, $d_{4}=2867 \mathrm{~nm}$

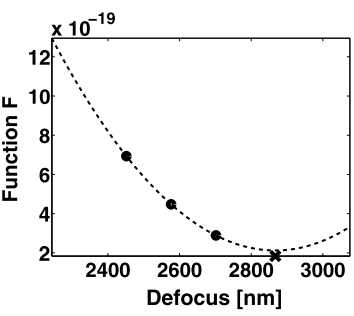

(r)

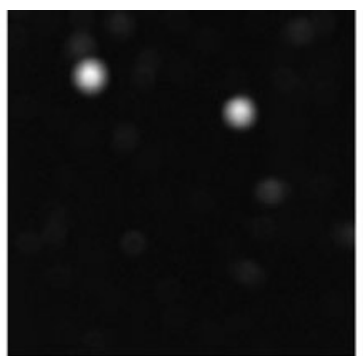

(c) $d_{1}=2184 \mathrm{~nm}$

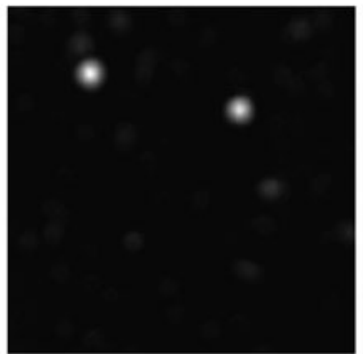

(g) $d_{2}=2384 \mathrm{~nm}$

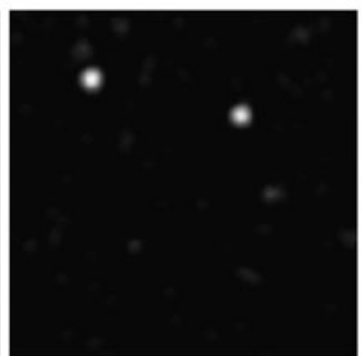

(k) $d_{3}=2584 \mathrm{~nm}$

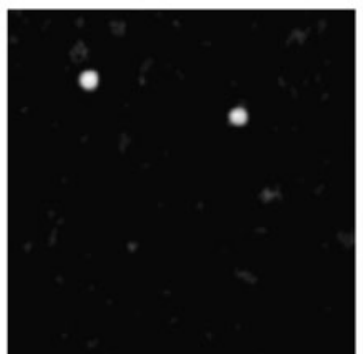

(o) Improved image, $d_{5}=2803 \mathrm{~nm}$

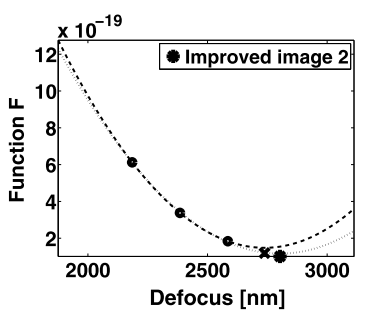

(s)

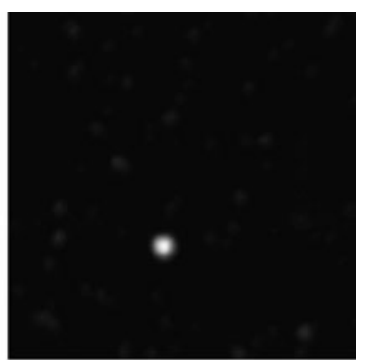

(d) $d_{1}=3322 \mathrm{~nm}$

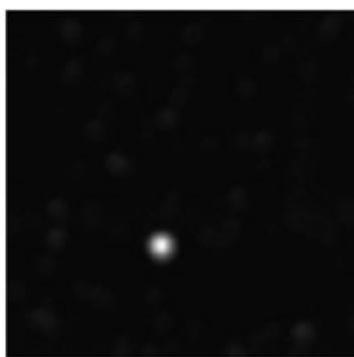

(h) $d_{2}=3522 \mathrm{~nm}$

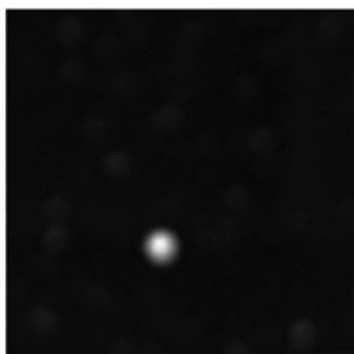

(l) $d_{3}=3722 \mathrm{~nm}$

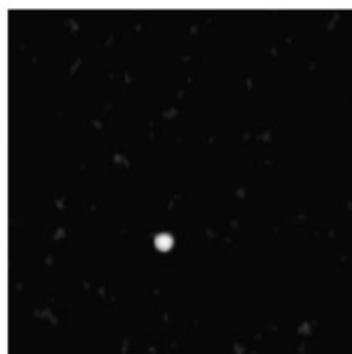

(p) Improved image, $d_{5}=3052 \mathrm{~nm}$

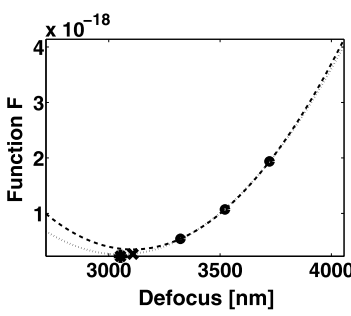

(t)

Fig. 10 On-line experiment: image defocus improvement via interpolation the function $F$ by a quadratic polynomial

position and magnification), as well as sample and environment instabilities. If the image is out-of-focus, the particle analysis software might give errors. For this reason it is im- portant to run the algorithm of automated defocus correction with a certain periodicity in time as a part of particle analysis automated application. 
Four examples of application runs are shown in Fig. 10. The images of gold particles are focused automatically. First two columns show autofocussing with three images, and the third and the fourth columns show autofocussing with four images. The recording of the fourth image might improve the final image quality (the function $F$ has a lower value). However, the improvement is not that strong. The difference between the fourth and the fifth resulting images in this experiment is not distinguishable by a human eye.

For all the runs of the method described in this section the value of $\alpha$ is fixed $\alpha=3$. The choice has been made experimentally by computing the function $F$ for experimental data and fitting it with a quadratic polynomial. The value of $\alpha$ corresponding to the smallest approximation error is chosen.

The values of parameter $\Delta d$ have been chosen experimentally for the particular application type, as well as the value of parameter $\alpha$. It is clear that $\Delta d$ would be larger than the tolerable defocus error, but not too large. In a realworld application $\Delta d$ should change proportionally to the magnification, as well as the tolerable defocus error changes proportionally to the magnification related to the pixel width value (see (27)). In the four experiments shown in Fig. 10, we have chosen $\Delta d=1000,125,200,200 \mathrm{~nm}$ correspondingly. The value of $\Delta d$ is adjusted automatically with the change in the magnification during the application runs.

\section{Discussion}

The new method for rapid autofocussing is developed and tested for the reference case of scanning transmission electron microscopy. The tests are performed with the standard calibration sample and the particle analysis application. The algorithm is based on general assumptions, and thus could be considered for other applications, such as electron tomography [37], as well as for other types of microscopes and different optical devices.

It has been proven that the derivative-based sharpness function is strictly monotone and has a unique optimum at the in-focus image for the noise-free image formation. This has already been used before on heuristic grounds in practical applications. The assumption of a Lévi point spread function is more general than a Gaussian point spread function used in a number of literature sources [4, 23, 42]. In a more general case the point spread function of a scanning transmission microscope could be modeled via the aberration function [13], which makes the model difficult for analysis. For a different point spread function model the optimum position of the sharpness function might change. For instance for the wave aberration-based point spread function, simulations show that the variance-based sharpness function reaches its optimum at the so-called Scherzer defocus [31], which is different from zero.
In the general case the sharpness function is not a quadratic polynomial. Nevertheless it has been shown that for the proper choice of the artificial blur $\alpha$, function $F$ can accurately be approximated by a quadratic polynomial. This provides the possibility of increasing the speed of the autofocus procedure. The digital image model applied for analysis in Sect. 4.2 could be replaced by a different model. In computer vision and computational photography for instance, it has been recently shown that the sharp image can accurately be described by a sparse function of image derivatives [17]. Intuitively, this technique could be also applied to microscopy images.

For our sharpness function we have chosen the $L^{2}$-norm, because it is the most practically used norm with a lot of proven mathematical properties. This simplifies the analysis. For instance a relatively trivial proof of the fact that derivative-based sharpness function reaches its maximum at $\sigma=0$ for the $L^{2}$-norm case could be complicated in the case of $L^{1}$-norm or the general $L^{p}$-norm. In practice the $L^{1}$ norm derivative-based sharpness function is used as well. The results of applying such a function to experimental data have been shown to be similar or worse than of the $L^{2}$ norm based function [18, 32, 40]. Our observations could be probably generalized for the $L^{p}$-norm case. However, we do not expect better results analytically nor numerically for the general $L^{p}$-norm.

The influence of noise, which is always present during the image formation in optical devices, on the sharpness function is not studied in this paper. We can see from numerical experiments with the real data as well as from the different papers $[18,31,33]$ that the noise in the image formation might result in the noise in the sharpness function, thus the function might obtain local optima and the statement of Property 1 is not true anymore. The noise sensitivity of different sharpness functions based on two error estimates has been studied in [33]. In our paper it is clear from numerical experiments with the real data and the on-line application runs that function $F$ is nearly a quadratic polynomial for a reasonable amount of noise (the machine settings for recording the images are chosen in the same way as for the real-world applications). The artificial blur parameter $\alpha$ provides image smoothing that results in the smoothing of the function. The quantification of the influence of noise and the automated optimal choice of parameter $\alpha$ could be a topic of a research study in future.

Astigmatism aberration of the optical device lens results in the point spread function, which is not rotationally symmetric. This phenomenon has not been studied in this paper, because we have considered only one-dimensional setting. The presence of the astigmatism aberration might result in the multiple optima in a sharpness function $[4,28]$. In one of the numerical experiments in this paper this effect has been shown. The influence of the astigmatism aberration on the 
sharpness function, and the stigmatic sharpness function improvement after applying the artificial blur have been studied in [30]. By considering two-dimensional case the method might be extended to the simultaneous automated defocus and astigmatism correction method.

Acknowledgements We kindly acknowledge R. Doornbos (ESI, The Netherlands) and S. Sluyterman (FEI, The Netherlands) for the recording of experimental data and on-line experiments, Wouter van den Broek (EMAT, Belgium) for providing experimental samples.

This work has been carried out as a part of the Condor project at FEI Company under the responsibilities of the Embedded Systems Institute (ESI). This project is partially supported by the Dutch Ministry of Economic Affairs under the BSIK program.

Open Access This article is distributed under the terms of the Creative Commons Attribution Noncommercial License which permits any noncommercial use, distribution, and reproduction in any medium, provided the original author(s) and source are credited.

\section{References}

1. Brenner, J.F., Dew, B.S., Horton, J.B., King, T., Neurath, P.W., Selles, W.D.: An automated microscope for cytologic research a preliminary evaluation. J. Histochem. Cytochem. 24(1), 100-111 (1976)

2. Carasso, A.S.: The APEX method in image sharpening and the use of low exponent levy stable laws. SIAM J. Appl. Math. 63(2), 593-618 (2002)

3. Carasso, A.S., Bright, D.S., Vladar, A.E.: APEX method and realtime blind deconvolution of scanning electron microscopy imagery. Opt. Eng. 41(10), 2499-2514 (2002)

4. Erasmus, S.J., Smith, K.C.A.: An automatic focusing and astigmatism correction system for the SEM and CTEM. J. Microsc. 127(2), 185-199 (1982)

5. Erteza, A.: Sharpness index and its application to focus control. Appl. Opt. 15(4), 877-881 (1976)

6. Goodhew, P.J., Humphreys, J., Beanland, R.: Electron Microscopy and Analysis, 3rd edn. Taylor \& Francis, London (2001)

7. Goodman, J.W.: Fourier Optics, 3rd edn. Roberts \& Company, Greenwood Village (2006)

8. Haider, M., Muller, H., Uhlemann, S.: Advances in imaging and electron physics. In: Present and Future Hexapole Aberration Correctors for High-Resolution Electron Microscopy, vol. 153, pp. 43-120. Academic Press, Amsterdam (2008)

9. Hilsenstein, V.: Robust autofocusing for automated microscopy imaging of fluorescently labelled bacteria. In: Proc. International Conference on Digital Image Computing: Techniques and Applications (2005)

10. Johnson, C.B.: A method for characterizing electro-optical device modulation transfer function. Photogr. Sci. Eng. 14, 413-415 (1970)

11. Jutamulia, S., Asakura, T., Bahuguna, R.D., De Guzman, C.: Autofocusing based on power-spectra analysis. Appl. Opt. 33(26), 6210-6212 (1994)

12. Kautsky, J., Flusser, J., Zitova, B., Simberova, S.: A new waveletbased measure of image focus. Pattern Recognit. Lett. 23, 17851794 (2002)

13. Kirkland, E.J.: Advanced Computing in Electron Microscopy. Plenum Press, New York (1998)

14. Krotkov Focusing, E.: Int. J. Comput. Vis. 1, 223-237 (1987)

15. Kumar, K., Pisarenco, M., Rudnaya, M.E., Savcenco, V., Srivastava, S.: Shape reconstruction techniques for optical sectioning of arbitrary objects. Math. Ind. Case Stud. J. 3, 19-36 (2011)
16. Le, T., Chartrand, R., Asaki, T.J.: A variational approach to reconstructing images corrupted by Poisson noise. J. Math. Imaging Vis. 27(3), 257-263 (2007)

17. Levin, A., Weiss, Y., Durand, F., Freeman, W.T.: Understanding and evaluating blind deconvolution algorithms. In: Proc. of IEEE Conf. on Computer Vision and Pattern Recognition (CVPR) (2009)

18. Liu, X.Y., Wang, W.H., Sun, Y.: Dynamic evaluation of autofocusing for automated microscopic analysis of blood smear and pap smear. J. Microsc. 227, 15-23 (2007)

19. Lupini, A.R., Pennycook, S.J.: Rapid autotuning for crystalline specimens from an inline hologram. J. Electron Microsc. 57(6), 195-201 (2008)

20. Morigi, S., Reichel, L., Sgallari, F., Shyshkov, A.: Cascadic multiresolution methods for image deblurring. SIAM J. Imaging Sci. 1(1), 51-74 (2007)

21. Muller, R.A., Buffington, A.: Real-time correction of atmospherically degraded telescope images through image sharpening. J. Opt. Soc. Am. 64(9), 1200-1210 (1974)

22. Nakamae, K., Chikahisa, M., Fujioka, H.: Estimation of electron probe profile from SEM image through wavelet multiresolution analysis for inline SEM inspection. Image Vis. Comput. 25, 11171123 (2007)

23. Nayar, S.K., Nakagawa, Y.: Shape from focus. IEEE Trans. Pattern Anal. Mach. Intell. 16(8), 824-831 (1994)

24. Ong, K.H., Phang, J.C.H., Thong, J.T.L.: A robust focusing and astigmatism correction method for the scanning electron microscope-part II: autocorrelation-based coarse focusing method. Scanning 20, 324-334 (1997)

25. Papoulis, A.: Signal Analysis. McGraw-Hill, New York (1977)

26. Rudnaya, M.E., Kho, S.C., Mattheij, R.M.M., Maubach, J.M.L.: Derivative-free optimization for autofocus and astigmatism correction in electron microscopy. In: Proc. 2nd International Conference on Engineering Optimization, Lisbon, Portugal (2010)

27. Rudnaya, M.E., Mattheij, R.M.M., Maubach, J.M.L.: Iterative autofocus algorithms for scanning electron microscopy. Microsc. Microanal. 15(2), 1108-1109 (2009)

28. Rudnaya, M.E., Mattheij, R.M.M., Maubach, J.M.L.: Evaluating sharpness functions for automated scanning electron microscopy. J. Microsc. 240, 38-49 (2010)

29. Rudnaya, M.E., Mattheij, R.M.M., Maubach, J.M.L., ter Morsche, H.: Autocorrelation-based sharpness functions. In: Proc. 3rd IEEE Int. Conf. on Sign. Proc. Syst., Yantai, China (2011)

30. Rudnaya, M.E., Mattheij, R.M.M., Maubach, J.M.L., ter Morsche, H.: Gradient-based sharpness function. In: Proc. International Conference of Applied and Engineering Mathematics, London, UK (2011)

31. Rudnaya, M.E., Van den Broek, W., Doornbos, R.M.P., Mattheij, R.M.M., Maubach, J.M.L.: Autofocus and twofold astigmatism correction in HAADF-STEM. Ultramicroscopy 111, 1043-1054, (2011)

32. Santos, A., De Solórzano, C.O., Vaquero, J.J., Peña, J.M., Malpica, N., Del Pozo, F.: Evaluation of autofocus functions in molecular cytogenetic analysis. J. Microsc. 188, 264-272 (1997)

33. Subbarao, M., Tyan, J.: Selecting the optimal focus measure for autofocusing and depth-from-focus. IEEE Trans. Pattern Anal. Mach. Intell. 20(8), 864-870 (1998)

34. Sun, Y., Duthaler, S., Nelson, B.J.: Autofocusing in computer microscopy: selecting the optimal focus algorithm. Microsc. Res. Tech. 65, 139-149 (2004)

35. Tanaka, N., Hu, J.J., Baba, N.: An on-linea correction method of defocus and astigmatism in HAADF-STEM. Ultramicroscopy 78, 103-110 (1999)

36. Tejada, A., Van Den Broek, W., van der Hoeven, S., den Dekker, A.J.: Towards STEM control: modeling framework and development of a sensor for defocus control. In: Proc. 48th IEEE Conference on Decision and Control, pp. 8310-8315 (2009) 
37. Van den Broek, W.: Advanced focus methods in electron microscopy: tomographic reconstruction of the EELS data cube autofocus of HAADF-STEM images. PhD thesis, University of Antwerp, 2007

38. Vollath, D.: Automatic focusing by correlative methods. J. Microsc. 147, 279-288 (1987)

39. Yang, G., Nelson, B.J.: Wavelet-based autofocusing and unsupervised segmentation of microscopic images. In: Proc. of the Intl. Conference on Intelligent Robots and Systems (2003)
40. Yeo, T.T.E., Ong, S.H., Jayasooriah, Sinniah, R.: Autofocusing for tissue microscopy. Image Vis. Comput. 11(10), 629-639 (1993)

41. Zemlin, F., Weiss, K., Schiske, W., Kunath, W., Herrmann, K.H.: Coma-free alignment of high resolution electron microscopes with the aid of optical diffractograms. Ultramicroscopy 3, 49-60 (1978)

42. Zhang, Y., Zhang, Y., Changyun, W.: A new focus measure method using moments. Image Vis. Comput. 18, 959-965 (2000) 